\title{
EVIDENCE OF HOMOLOGOUS AND HETEROLOGOUS EFFECTS AFTER UNILATERAL LEG TRAINING IN YOUTH
}

\begin{tabular}{|r|l|}
\hline Journal: & Applied Physiology, Nutrition, and Metabolism \\
\hline Manuscript ID & apnm-2017-0338.R2 \\
\hline Manuscript Type: & Article \\
\hline Date Submitted by the Author: & 20 -Sep-2017 \\
\hline Complete List of Authors: & $\begin{array}{l}\text { Ben Othman, Aymen; Centre National de la Medecine et des Sciences du } \\
\text { sport } \\
\text { Behm, David; Memorial University of Newfoundland, } \\
\text { Chaouachi, Anis; Centre National de la Medecine et des Sciences du sport }\end{array}$ \\
\hline $\begin{array}{r}\text { Is the invited manuscript for } \\
\text { consideration in a Special } \\
\text { Issue? : }\end{array}$ & \\
\hline Keyword: & $\begin{array}{l}\text { pediatrics < pediatrics, strength training < exercise, power output < } \\
\text { exercise, resistance training < exercise, cross education }\end{array}$ \\
\hline
\end{tabular}


AUTHORS: Aymen Ben Othman ${ }^{1}$, David G Behm ${ }^{3}$, Anis Chaouachi ${ }^{1,2}$

CORRESPONDING AUTHOR: David G. Behm School of Human Kinetics and Recreation, Memorial University of Newfoundland, St. John's, Newfoundland, Canada, A1C 5S7 dbehm@mun.ca Tel: 709-864-3408

Fax : 709-864-3979

\section{RUNNING TITLE:}

3

4

5




\section{ABSTRACT}

The positive effects of unilateral training on contralateral muscles (cross education) has been

31

32 demonstrated with adults for over a century. There is limited evidence for cross education of heterologous muscles. Cross education has not been demonstrated with children. It was the objective of this study to investigate cross education training in children examining ipsilateral and contralateral homologous and heterologous muscles. Forty-eight male children (10-13 years) were assessed for unilateral, ipsilateral and contralateral lower limb strength, power and endurance (1 repetition maximum (RM) leg press, knee extensors (KE) and flexors (KF) maximum voluntary isometric contractions (MVIC), countermovement jump (CMJ), muscle endurance test (leg press repetitions with 60\% 1RM)), and upper body unilateral MVIC elbow flexors (EF) and handgrip strength. An eight-week training program involved two unilateral leg press resistance training groups (high load-low repetitions: 4-8 sets of 5RM, and low load-high repetitions:1-2 sets of 20RM) and control (untrained) group. All muscles exhibited improvements of $6.1 \%$ to $89.1 \%$. The trained limb exhibited greater adaptations than the untrained limb for leg press 1 RM (40.3\% vs. $25.2 \%$; $p=0.005)$, and 60\% 1 RM leg press (104.1\% vs. $73.4 \%$; $\mathrm{p}=0.0001)$. The high load-low repetition training induced $(\mathrm{p}<0.0001)$ greater improvements than low load-high repetition with KE, KF, EF MVIC and leg press 1RM. This is the first study to demonstrate cross education effects with children and the effects of unilateral training involve both contralateral homologous and heterologous muscles with the greatest strength training responses from high load-low repetition training.

KEY WORDS: cross education; pediatric; strength training; power output; resistance training; 
Evidence for cross education was first published over a century ago (Scripture 1894).

53 Cross education involves the performance improvement (i.e., strength, skill execution,

54 endurance) of the untrained limb after a period of unilateral practice (i.e., strength training, skill

55 learning, endurance training) (Farthing et al. 2007). Ample evidence for cross education has been

56 demonstrated in adults for contralateral homologous muscles such as the quadriceps (Goodwill

57 and Kidgell 2012, Kannus et al. 1992; Hortobagyi et al. 1997; Evetovich et al. 2001; Latella et al.

58 2012), elbow flexors (EF) (Ebersole et al. 2002; Adamson et al. 2008), and handgrip muscles

59 (Shields et al. 1999). Strength increases in the contralateral limb following unilateral strength

60 training of the ipsilateral limb has provided non-significant (Farthing and Chilibeck 2003), small

61 (i.e. 3.8-5\%) (Housh and Housh 1993; Munn et al. 2005) and very large improvements (i.e. 35\%,

$6252 \%, 77 \%$ ) (Goodwill and Kidgell 2012; Hortobagyi et al. 1997). The magnitude of adaptation

63 depends on the characteristics of the intervention (e.g. different training protocols, eccentric or

64 concentric contractions) performed (Cirer-Sastre 2017), trained state of the participant (sedentary

65 or athlete), and the involved muscle groups (Hortobagyi 2005).

Surprisingly in the last century, cross education has not been examined with children.

67 Since neuromuscular responses to strength training can differ between children and adults

68 (Ramsay et al. 1990; Behm et al. 2008), it is not known whether this phenomenon is evident in

69 children. It could be speculated that since children's strength gains are predominantly ascribed to

70 neural adaptations (Ozmun et al. 1994; Faigenbaum et al. 1996; Falk et al. 1996; Faigenbaum

71 2000; Behm et al. 2008; Faigenbaum et al. 2009), their cross education response may exceed

72 adults. While adults also experience neural adaptations (Behm 1995, Kidgell et al. 2011, Latella 
73 et al. 2012), they typically can access greater morphological (i.e. muscle hypertrophy) training

74 adaptations which would not be expected to transfer to non-exercised muscles (Behm 1995).

In adults, a low repetition/high load RT program is recommended to increase maximal

76

77

78

79

80

81

82

83

84

85

86

87

88

89 strength and high repetition/low load program to increase muscular endurance (Behm 1995).

Other adult studies have examined cross education effects showing a proportional relationship between the applied training load and the effect achieved (i.e. high loads or intensities increase muscle strength; low loads or intensities with high repetitions increase muscle endurance) (Kannus et al. 1992; Ebersole et al. 2002; Housh et al. 1992; Housh and Housh 1993; Evetovich et al. 2001; Farthing and Chilibeck 2003). However, in 5 to 12-year-old children, Faigenbaum et al. (1999) demonstrated that high repetition/low load and low repetition/high load resistance training programs resulted in similar enhancements of maximal strength. Manipulation of the various acute resistance training variables (e.g., number of sets, repetitions, intensity, or load) are believed to influence the magnitude of strength adaptations on the trained limb (Behm 1995), and could also affect adaptations of contralateral strength (Munn et al. 2005). It is not known whether high or lower loads or intensity resistance training_would similarly affect cross education or global training effects with dynamic and isometric strength, power (i.e. countermovement jumps (CMJ)) or muscle endurance with children.

According to Hortobagyi's review (2005) cross education is specific to the homologous muscle pair. However, Sariyildiz et al. (2011) did show a cross education training effect to a heterologous muscle. While there are some examples of acute non-local fatigue and stretching effects upon heterologous muscle groups (Halperin et al. 2014; Behm et al. 2016; Sambaher et al. 2016; Aboodarda et al. 2017), there is very little literature monitoring the extent of cross 
95 education effects to heterologous muscles and no information regarding youth. Greater global or 96 cross education responses with children would provide a rationale for a robust application of

97 these techniques when rehabilitating unilateral injuries by training the contralateral limb. 98 Furthermore, in youth athletic populations, the prevention techniques of unilateral overuse 99 injuries in sports such as baseball, softball, racquet sports and others could be implemented by 100 training the contralateral limb, decreasing the workload on the dominant over-exercised limb.

101

102 103

Thus, due to the absence of studies comparing the effects of different variables for equalvolume unilateral traditional resistance training in children, the aim of this study was to compare the effects of two-equal-volume unilateral resistance training programs (high load/low repetition vs. low load/high repetition) on maximal dynamic and isometric lower body muscle strength, endurance, unilateral vertical jump performance, handgrip strength and unilateral elbow flexors maximal voluntary isometric contractions (MVIC). Since the literature indicates that both cross education (Lee et al. 2007; Farthing 2009)_and youth resistance training (Ozmun et al. 1994; Faigenbaum et al. 1996; Falk and Tenenbaum 1996; Faigenbaum 2000; Behm et al. 2008; Faigenbaum et al. 2009) have a high neural component, it is hypothesized that cross education effects in this population will be readily apparent in a variety of muscles and tasks.

\section{MATERIALS AND METHODS}

\section{Participants}

Forty-eight healthy male children between 10-13 years, recruited from the same public school of Bou-Arada city, Tunisia, volunteered to participate in this study (Table 1). All participants were from similar socio-economic status and had the same daily school schedules. 
116 All of them participated in their normal physical education lessons and none were involved in

117 any after-school activities or any formalized strength and conditioning training program. A

118 maturity status assessment was conducted using the noninvasive technique proposed by Mirwald

119 et al. (2002). All participants were classified at the pre-peak height velocity stage of physical

120 maturation (Table 1). Parental and subject informed consent was obtained after thorough

121 explanation of the objectives and scope of this project, the procedures, risks, and benefits of the

122 study. The study was conducted according to the Declaration of Helsinki and the protocol was

123 fully approved by the Ethics Committee of the National Centre of Medicine and Science of

124 Sports of Tunis (CNMSS) before the commencement of the assessments. Subjects and their

125 parent/guardian were also informed that participation was voluntary and that they could

126 withdraw from the study at any time. None of the participants had any history of

127 musculoskeletal, neurological or orthopedic disorders that might impair their ability to execute

128 resistance exercise training or to perform strength and power tests.

129

130

131

132

133

134

135

136

137

Experimental procedures

One week before the commencement of the study, all subjects participated in three orientation sessions to become familiar with the general environment, form and technique on each test used to evaluate force and power, technique for each training exercise, equipment, and the experimental procedures to minimize the learning effect during the course of the study. Each subject's height and body mass were collected using a wall-mounted stadiometer (Easy Glide Stadiometer Perspective Enterprises, Portage, Michigan) and electronic scale (LifeSource Model UC-321P, made by A\&D Company, Tokyo, Japan), respectively. Afterwards, participants' performances were tested pre- and post- the 8-week unilateral training period. The testing 
138 protocol included assessment of lower limb unilateral strength and power (1RM horizontal leg 139 press, maximum voluntary isometric contraction (MVIC) of quadriceps and hamstring muscles, 140 countermovement jump (CMJ), muscle endurance test (maximum number of leg press repetitions 141 with 60\% 1RM)), and upper body unilateral MVIC strength of EF and hand grip strength. 142 Following the initial baseline testing session, subjects were randomly divided into two unilateral 143 resistance training groups (high load/low repetitions, $\mathrm{n}=16$ and low load/high repetitions group $144 \mathrm{n}=16)$ and a control group $(\mathrm{n}=16)$ without a training program. Using a controlled randomization 145 method, groups were matched for age, maturation status and physical characteristics. The high 146 load/low repetition group performed 4 to 8 sets of 5 repetitions maximum (RM) of unilateral 147 seated leg press (knee and hip extension/flexion) with 2 min rest interval between sets. The low $148 \mathrm{load} /$ high repetition group performed 1 to 2 sets of $20 \mathrm{RM}$ with $2 \mathrm{~min}$ rest interval between sets. 149 The volume of work during training was equal.

\section{Training programs}

The participants trained for 8 weeks, completing three sessions per week with at least 48- 72

152 hours of rest between sessions (totaling 24 sessions). Since with the testing periods, the duration

153 of the experiment was 10 weeks, an 8-week training period fit into the school semester period of 154 the participants. Subjects were randomly assigned to one of two training groups: unilateral high 155 load-low leg press repetition group, unilateral low load-high repetition leg press group or the 156 control group (untrained). The high load/low repetitions training group performed 4 (week 1 and 157 8) or 8 sets (weeks 2-7) of 5 repetitions with 2 minutes of recovery between sets. The low 158 load/high repetitions training group was subjected to 1 (week 1 and 8) or 2 sets (weeks 2-7) of 20 159 repetitions with 2 minutes of recovery between sets. Progressive overload was implemented by 
160 increasing the load by $5-10 \%$ whenever a participant could exceed the prescribed 5 or 20 161 repetitions. The control group was limited to their regularly scheduled physical education class.

162 163 164 165 166 167 168 169 170 171

172 beyond the prescribed training zone, the weight was increased to bring the number of repetitions 173 back within the RM training zone. Two minutes recovery was allowed between sets for both 174 training programs.

175 176

All participants complied_with these instructions. Prior to each training session, the training groups underwent a 5-min warm-up including submaximal ergometer cycling and dynamic stretching. The exercise used for training was a unilateral leg press with the dominant leg in a seated position using a commercial horizontal leg press (Life Fitness Pro Horizontal Leg Press) with a range of motion 90 to $10^{\circ}\left(0^{\circ}=\right.$ full knee extension). Participants in the training groups received skill-specific feedback on the quality of each movement and they were informed to never lock the knee on horizontal leg press during training or testing. The instructors recorded the training data on workout logs daily and made appropriate adjustments in training resistance and repetitions. Special attention was paid to the instructions to keep the opposite leg completely immobile and as relaxed as possible during the training. If the subject performed repetitions training programs.

\section{Lower body maximal strength and muscular endurance tests}

Unilateral leg press maximal dynamic strength (1 RM):

Unilateral leg strength was assessed on the horizontal leg press with a one-repetition maximum (1-RM) test as reported by Faigenbaum et al. (2003). Before attempting a 1RM, participants performed 3 submaximal sets of 1-6 repetitions with a light to moderate load, then 3 sets of a heavier load. Finally, participants performed a series of single repetitions with increasing loads. If the weight was lifted with the proper form, it was increased by approximately 
182 183

184

185

186

187

188

189

190

191 192 subjects.

193

194

195

196

197

198

199

200

201

202

1-2 kg, and the participant_attempted another repetition. The increments in weight were dependent on the effort required for the lift and became progressively smaller as the subject reached 1RM. 1RM was defined as the greatest load lifted through a full range of motion (ROM) before 2 failed attempts at a given load. The exercise execution technique was standardized and continuously monitored in an attempt to assure the quality of the data. The participants were strapped into the apparatus with a seatbelt with the non-exercised leg positioned off the leg press apparatus (foot on the floor) in a relaxed state. Participants folded their arms across their chest during the procedure. Failure was defined as a lift falling short of the full ROM on at least 2 attempts spaced at least 2 minutes apart. Throughout all testing procedures, an instructor-tosubject ratio of 1:1 was maintained, and uniform verbal encouragement was offered to all

\section{Unilateral leg press muscular endurance test}

Once the 1RM was determined, $60 \%$ of this value was calculated for the local muscular endurance test. Subjects were asked to perform the horizontal leg press for as many repetitions as possible with $60 \%$ of 1RM until failure (Radaelli et al. 2015).

\section{Unilateral isometric strength (Quadriceps and Hamstring MVIC)}

Maximal isometric knee extensor and knee flexors strength were measured in both the dominant (trained) and non-dominant (untrained) limbs using a calibrated hand held dynamometer (Microfet 2; Hogan Health Industries Inc., Draper, Utah, USA). Specifics of the test position, stabilization, and dynamometer placement used in this study were chosen according to the instrument manual instructions as previously described (Chaouachi et al. 2017). The 
203 dynamometer incorporates a load cell and has a digital display. The hand-held dynamometer was

204 placed perpendicular to the anterior aspect of the tibia, just proximal of the medial malleolus for

205 quadriceps testing and against the Achilles tendon for hamstrings testing. For quadriceps testing,

206 participants were seated on the chair of leg extension machine, positioned so that both feet were

207 off the ground, with hips and knees both flexed at $90^{\circ}$. The lever arm of the leg extension

208 machine was fixed at $100^{\circ}$. The dynamometer was fixed and stabilized by the examiner between

209 the lever arms of the machine and the specific testing placement position on the tested limb

210 segment. The arm of the leg extension machine was fixed with a maximal load to ensure that

211 participants performed an isometric contraction. Participants were instructed to exert maximal

212 force against the dynamometer for a period of three to five seconds while the dynamometer was

213 held stationary. Three consecutive trials separated by approximately 1-min for both legs and the

214 highest values were recorded for analysis. The same procedure and instructions were utilized to

215 measure the MVIC of hamstring muscles. Hamstring MVIC testing was performed with the

216 subjects in a prone position on a leg flexion machine with hips in a neutral extension and knees

217 flexed to $90^{\circ}$. High hand-held dynamometry reliability measurements in similar pediatric

218 population in our laboratory have been reported elsewhere (Chaouachi et al. 2017).

\section{Unilateral Countermovement Jump (CMJ)}

The unilateral CMJ test was performed using an Ergo Jump system (Ergojump: Globus

221 Italia, Codogne, Italy) according to the procedure described by_Chaouachi et al. (2014).

222 Participant started from an upright akimbo (hands on hip) position. Participants were encouraged

223 to perform the eccentric phase of the jump as quickly as possible to maximize jump height.

224 Participant self-selected the amplitude of the knee flexion of the CMJ to avoid changes in the 
225 coordination pattern. The non-jumping leg was held in a slightly flexed relaxed position during

226 the unilateral jump. Three trials were performed for each leg with approximately 1-min of

227 recovery between trials and the highest jump was used for analysis.

228 Upper body maximal voluntary isometric contraction (MVIC)

229 Unilateral elbow flexors (EF) MVIC

EF MVIC strength of both arms was measured using a calibrated hand held dynamometer

231 (Microfet 2; Hoggan Health Industries Inc., Draper, Utah, USA) as previously described (Ben

232 Othman et al. 2017). The hand-held dynamometer was placed between the flexor aspect of the

233 wrist and the lever of elbow flexion machine to ensure an isometric contraction. The elbow

234 flexed at $90^{\circ}$, and then the participant exerted a 3-5 second MVIC against the dynamometer

235 placed perpendicularly against the forearm. This procedure was repeated three times for both the

236 right and left hands with an approximate 1-min rest period and the highest value was recorded

237 for analysis. High reliability of this test in similar pediatric population in our laboratory has been

238 reported elsewhere (Ben Othman et al. 2017).

\section{Unilateral Handgrip strength}

MVIC handgrip strength $(\mathrm{kg})$ was measured using a calibrated hand dynamometer (Takei,

241 Tokyo, Japan) as previously described (Ben Othman et al. 2017). Participants stood comfortably

242 with the arm adducted at approximately $45^{\circ}$. The dynamometer was held freely without support

243 and did not touch the subject's trunk, with constant extension of the elbow. The grip-span of the

244 dynamometer was adjusted to each participant's hand size so that the proximal inter-phalangeal

245 joints of the 4 fingers rested on one side of the handgrip and that of the thumb rested on the other 
246 side. Participants were required to exert MVIC strength on the dynamometer. Three trials

247 separated by an approximate 1 min rest interval for each hand were performed, and the maximum

248 score for each hand was recorded. Excellent MVIC handgrip strength reliability measurements in

249 children in our laboratory have been reported elsewhere (Ben Othman et al. 2017).

Statistical Analysis

251

252

253

254

255

256

257

258

259

260

261

262

263

264

265

266

267

268

Statistical analyses were computed using SPSS software (Version 22.0, SPSS, Inc.,

Chicago, IL). Dependent variables underwent assumption of normality (Shapiro-Wilk test) and sphericity (Mauchley test), and when violated, the corrected value for non-sphericity with

Greenhouse-Geisser Epsilon was reported. A three-way repeated measures ANOVA (2x3x2) was performed for each measure to determine the existence of significant differences between trained (dominant) and untrained (non-dominant) limbs, type of training (high load/low repetitions, low load/high repetitions and control) and time (pre- and post-training). An alpha level of $\mathrm{p}=0.05$ was considered statistically significant. If significant main effects were demonstrated, Bonferroni post hoc analysis were conducted when appropriate. Independent t-tests were used to assess simple effects of interacting main variables. Absolute mean changes as well as contralateral homologous (cross education) and global (contralateral and ipsilateral heterologous untrained muscles) strength transfer values are reported. Contralateral strength transfer was calculated by determining the difference in changes of the experimental (high load/low repetitions, low load/high repetitions) and control groups pre- to post-training (Carroll et al. 2006, Kidgell et al. 2011). The calculation was performed as follows:

$$
\left.\frac{(\text { Exp-post-Exp-pre })-(\text { Control-post }- \text { Control-pre }}{}\right)^{* 100} \text { Control-pre }
$$


269 Exp-post and Exp-pre refer to the pre- and post-test mean scores of the untrained arm for either 270 of the experimental groups (high load/low repetitions, low load/high repetitions) while Control271 pre and Control-post refer to the pre- and post-test mean scores of the control group. Relative 272 (percentage) changes as well as the magnitude of change was calculated and reported as trivial $273(<0.2)$, small (0.2-0.49), medium (0.5-0.79) or large $(\geq 0.8)$ effect sizes (d) (Cohen 1988).

274 Reliability was calculated with Cronbach alpha interclass correlation coefficient (ICC).

275 Descriptive statistics include means \pm standard deviations (SD).

\section{RESULTS}

277 Reliability

279 groups (Table 2).

280 Training Effects

Every measure (note: all measures were unilateral) expressed a significant main effect for

282 time with pre- to post-training improvements ranging from 5.6\% $\left(\mathrm{F}_{(1,15)}=20.77 ; \mathrm{p}<0.0001\right.$;

$283 \mathrm{~d}=0.58$; elbow flexors MVIC $), 8.6 \%\left(\mathrm{~F}_{(1,15)}=36.0 ; \mathrm{p}<0.0001 ; \mathrm{d}=0.71 ;\right.$ handgrip $), 18.2 \%\left(\mathrm{~F}_{(1,15)}=\right.$ $284122.49 ; \mathrm{p}<0.0001 ; \mathrm{d}=0.89 ; \mathrm{CMJ}), 18.9 \%\left(\mathrm{~F}_{(1,15)}=139.47 ; \mathrm{p}<0.0001 ; \mathrm{d}=0.90 ;\right.$ knee flexors 285 MVIC $), 28.2 \%\left(\mathrm{~F}_{(1,15)}=281.87 ; \mathrm{p}<0.0001 ; \mathrm{d}=0.95\right.$; knee extensors MVIC $), 32.7 \%\left(\mathrm{~F}_{(1,15)}=\right.$ $286149.39 ; \mathrm{p}<0.0001 ; \mathrm{d}=0.91 ;$ leg press $1 \mathrm{RM})$ to $89.1 \%\left(\mathrm{~F}_{(1,15)}=299.23 ; \mathrm{p}<0.0001 ; \mathrm{d}=0.95 ; 60 \%\right.$

287 1RM leg press) overall. 
Except for handgrip, all measures showed a significant main effect for type of training

290

291

292

293

294

295

296

297

298

299

300

301

302

303

304

305

306

307

308

309

310

(elbow flexors MVIC; $\mathrm{F}_{(2,14)}=3.92 ; \mathrm{p}<0.0001 ; \mathrm{d}=0.21, \mathrm{CMJ} ; \mathrm{F}_{(2,14)}=3.08 ; \mathrm{p}<0.0001 ; \mathrm{d}=0.27$,

knee flexors MVIC; $\mathrm{F}_{(2,14)}=17.35 ; \mathrm{p}<0.0001 ; \mathrm{d}=0.54$, knee extensors $\mathrm{MVIC} ; \mathrm{F}_{(2,14)}=18.25$;

$\mathrm{p}<0.0001 ; \mathrm{d}=0.55$, leg press $1 \mathrm{RM} \mathrm{F}_{(2,14)}=3.21 ; \mathrm{p}<0.0001 ; \mathrm{d}=0.28,60 \% 1 \mathrm{RM}$ leg press $\mathrm{F}_{(2,14)}=$

26.16; $\mathrm{p}<0.0001 ; \mathrm{d}=0.64)$. Only for knee flexors MVIC did the high load/low repetition trained

group provide a higher measure than the low load/high repetition training by $8.7 \%(\mathrm{p}=0.02)$. The

low load/high repetition trained group did not show any advantages over the high load/low

repetition training for any measure. The high load/low repetition trained group measures

exceeded the control group with every measure by $24.3 \%(p<0.0001$; knee extensors MVIC),

20.9\% ( $<<0.0001$; knee flexors MVIC), 11.2\% ( $\mathrm{p}=0.01$; elbow flexors MVIC), $17.6 \%$ ( $\mathrm{p}=0.05$

handgrip), $19.1 \%(p=0.05 ; C M J), 25.6 \%(p=0.05 ;$ leg press $1 \mathrm{RM})$ and $44.1 \%(p<0.0001 ; 60 \%$

1RM leg press). The low load/high repetition training did not significantly differ from control for

EF MVIC and leg press 1RM. However, the low load/high repetition training was significantly

greater than control with the knee extensors MVIC $(18.1 \%$; $<<0.0001)$, knee flexors MVIC

(11.7\%; $\mathrm{p}=0.03), \mathrm{CMJ}(5.3 \% ; \mathrm{p}=0.05)$, and 60\% 1RM leg press $(57.2 \% ; \mathrm{p}<0.0001 ; 60 \%)$.

The pre- to post-training improvements with the high load/low repetition group measures were substantially greater than low load/high repetition (significant interactions) with knee extensors MVIC (55.1\% vs. 29.4\%; p<0.0001), knee flexors MVIC (36.2\% vs. $18.1 \%$;

$\mathrm{p}<0.0001)$, EF MVIC (14.1\% vs. 3.3\%; p<0.0001), and leg press 1RM (64.5\% vs. 32.9\%;

$\mathrm{p}<0.0001)$. Although all training improvements were significant, there were no significant differences with pre- to post-training measures of low load/high repetition versus high load/low repetition measures for handgrip (16.1\% vs. 7.5\%), CMJ (38.4\% vs. 23.1\%) and 60\% 1RM leg 
311 press (148.4\% vs. 117.1\%). Table 3 illustrates mean absolute vs. contralateral or global strength

312 transfer percentage changes.

313 Trained vs. Untrained Limbs

With the exception of CMJ, every measure demonstrated a significant main effect for

315 trained vs untrained limbs. Overall, the dominant trained leg exceeded the non-dominant

316 untrained leg by $6.7 \%\left(\mathrm{~F}_{(1,15)}=25.32 ; \mathrm{p}<0.0001 ; \mathrm{d}=0.63\right.$; knee extensors $\left.\mathrm{MVC}\right), 3.5 \%\left(\mathrm{~F}_{(1,15)}=\right.$

317 12.93; $\mathrm{p}=0.003 ; \mathrm{d}=0.46 ;$ knee flexors MVC $), 6.9 \%\left(\mathrm{~F}_{(1,15)}=25.03 ; \mathrm{p}<0.0001 ; \mathrm{d}=0.63 ;\right.$ leg press

$3181 \mathrm{RM})$ and $17.0 \%\left(\mathrm{~F}_{(1,15)}=67.4 ; \mathrm{p}<0.0001 ; \mathrm{d}=0.82 ; 60 \% 1 \mathrm{RM}\right.$ leg press $)$. Since the upper body

319 was not trained, these measures compared untrained dominant (ipsilateral) versus untrained, non-

320 dominant (contralateral) changes. The dominant side showed 5.7\% and 3.3\% significantly

321 greater values for handgrip $\left(\mathrm{F}_{(1,15)}=19.26 ; \mathrm{p}=0.001 ; \mathrm{d}=0.56\right)$ and $\operatorname{EF} \operatorname{MVIC}\left(\mathrm{F}_{(1,15)}=22.41\right.$;

$322 \mathrm{p}<0.0001 ; \mathrm{d}=0.60)$ respectively.

Although both the trained and untrained limbs demonstrated positive training adaptations

324 (trained limb $x$ time interactions), the trained limb exhibited greater training adaptations than the 325 untrained limb only for CMJ, leg press 1RM, and 60\% 1RM leg press. With the difference 326 approaching significance for the $\mathrm{CMJ}(\mathrm{p}=0.06)$, the trained leg had $20.2 \%$ improvements 327 compared to $16.3 \%$ increases for the untrained limb. For the leg press 1RM and 60\% 1RM leg 328 press, trained leg adaptations were $40.3 \%$ vs. $25.2 \%\left(\mathrm{~F}_{(2,14)}=58.95 ; \mathrm{p}=0.005 ; \mathrm{d}=0.80\right)$ and $329104.1 \%$ vs. $73.4 \%\left(\mathrm{~F}_{(2,14)}=48.86 ; \mathrm{p}<0.0001 ; \mathrm{d}=0.76\right)$ significantly greater compared to the 330 untrained leg respectively. There were no significant differences between the trained dominant 331 and untrained non-dominant KE and KF MVIC forces. In addition, there was no significant 
332 difference between untrained ipsilateral and contralateral handgrip and EF MVIC strength 333 measures.

334

335

336

337

338

339

340

341 under the high load-low repetition training program, the linear regression was $\mathrm{R}^{2}=0.88$ with

$35230.5 \%$ relatively higher training increases with the trained $(53.4 \pm 50.6 \%)$ versus untrained $(37.1$

$353 \pm 25.5 \%$ ) limbs. For the low load - high repetition training adaptations the overall linear 
354 regression was $\mathrm{R}^{2}=0.98$ with $17.9 \%$ relatively higher training increases with the trained $(47.8 \pm$ $355 \quad 51.7 \%)$ versus untrained $(40.5 \pm 41.3 \%)$ limbs.

Three way interactions are illustrated with figures 1-7.

\section{DISCUSSION}

The most important findings in the present study were 1) the evidence for cross education training adaptations in adolescent male youth, 2) that the untrained limb had relatively similar training adaptations as the trained limb for KE and KF MVIC, 3) non-local untrained muscles such as the handgrip and EF MVICs had similar dominant (ipsilateral) versus non-dominant (contralateral) increases in force output, and 4) greater strength training responses with the high load/low repetition group versus the low load/high repetition training.

To our knowledge, this is the first study to examine cross education adaptations with youth. Since Scripture's first description in 1894 (Scripture 1894), all studies have examined adult contralateral_homologous (non-trained) adaptations (Hortobagyi 2005). The relative extent of contralateral training adaptations is very diverse in the adult literature with trained vs. nontrained limb improvements ranging from $46 \%$ vs. 9.2\% for knee extension (Hortobagyi et al. 1992; Housh and Housh 1993; Housh et al. 1998), 41\% vs. 35\% for unilateral squat (Goodwill and Kidgell 2012), 20.2\% vs. 5\% for knee flexion (Housh and Housh 1993; Housh et al. 1998),

371 and 48\% vs. 1.2\% (non-significant) for elbow flexion (Housh and Housh 1993; Ebersole et al.

372 2002; Farthing and Chilibeck 2003; Munn et al. 2005, Kidgell et al. 2011) strength respectively.

373 Hortobagyi (2005) indicated that the magnitude of cross education is typically about $25 \%$, but in

374 some cases, force or skill transfer can reach $80 \%$. The transfers in the present study of youth 375 generally correspond to the aforementioned adult values. Similar relative (but not absolute) 
376 strength training gains are found with adults and youth in trained muscle groups (Behm et al.

377 2008). The similarities in the extent of global or cross education training effects suggest the

378 possibility of similar mechanisms (neural and motor learning adaptations).

In the present study, youth trained vs. non-trained leg improvements were significantly

greater for the trained leg for CMJ, leg press 1RM and 60\% 1RM leg press. On the contrary, there were no significant training differences with trained versus untrained KE, KF, EF MVICs or handgrip forces. There are also examples of non-significant differences in the training increases of contralateral homologous trained vs. untrained limbs in adults with 6,7 , and 8 weeks of training of isometric ulnar deviation MVIC (Farthing et al. 2005, 2007), isometric and isokinetic knee extension and flexion (Kannus et al. 1992), and EF MVIC (Adamson et al. 2008) respectively. With limited evidence for extensive pediatric muscle hypertrophy (Blimkie 1989;

387 Faigenbaum et al. 1996; 2009; Falk and Tenenbaum 1996; Behm et al. 2008), and youth are generally less experienced or skillful in most tasks than adults, the suggestion of a strong contribution of neural adaptations (typically suggested to be increased motor unit recruitment and rate coding) with youth strength training is ubiquitously emphasized in the literature (Blimkie 1989; Ramsay et al. 1990; Ozmun et al. 1994; Faigenbaum et al. 1996; 2009;

392 Faigenbaum 1996; Falk and Tenenbaum 1996; Hortobagyi 2005; Behm et al. 2008; Sale 1996).

393 More recent adult research employing transcranial magnetic stimulation have reported increases 394 in corticospinal excitability of the contralateral biceps brachii (Kidgell et al. 2011) as well as 395 decreased corticospinal inhibition of the contralateral quadriceps (Latella et al. 2012).

397 experience greater adaptations due to greater co-ordination and learning effects (Behringer et al. 398 2011; Behm et al. 2017), whereas trained youth may emphasize more neural (recruitment, and 
399

400

401

402

403

404

405

406

407

408

409

410

411

412

413

414

415

416

417

418

419

420

421

rate coding) and morphological adaptations (Behm et al. 2017). However, the possibility of global morphological adaptations (i.e. EF, handgrip) such as untrained muscle hypertrophic responses to the unilateral leg press training program is unlikely as Beyer et al. (2016) reported no increases in contralateral lower limb muscle size or chronic hormonal profile changes following a 4-week unilateral lower body and bilateral upper body resistance training program. The present study could not ascertain whether the similar homologous cross education training effects of adults and youth could have a proportionally greater contribution from motor learning adaptations with youth.

Although Hortobagyi (2005) stated that cross education is specific to the homologous muscle, the dynamic leg press training program provided significant improvements for all the tested homologous and heterologous muscle groups and exercises whether tested with isometric or dynamic contractions. Only one adult unilateral training study (Sariyildiz et al. 2011) investigated heterologous muscle strength gains (trained wrist flexors and tested both wrist flexors and extensors). Acute adult studies have shown non-local heterologous muscle fatigue (Halperin et al. 2014; Sambaher et al. 2016; Aboodarda et al. 2015, 2017) and stretching effects (Behm et al. 2016). Ben Othman et al. (2017) induced acute unilateral KE fatigue in 10-13 year old children showing non-local muscle effects both ipsilaterally (handgrip, EF MVIC, unilateral CMJ, unilateral balance) and contralaterally (handgrip, KE MVIC, KE isokinetic torque, unilateral CMJ, unilateral balance). In agreement with training specificity (Behm and Sale 1993), the significant disparity in training improvements of the trained and untrained legs only occurred with testing measures that either exactly (leg press) or somewhat resembled (CMJ) the training exercise (leg press). However, there were no significant differences in training improvements between similar muscle groups for non-specific tests (KE, KF MVIC: isometric tests vs. 
422 isoinertial training, uniarticular tests vs. biarticular training) or disparate, non-local, untrained,

423 muscles for non-specific tests (EF MVIC, handgrip MVIC). It can be surmised that motor

424 control, learning, coordination and perhaps some morphological changes were enhanced to a

425 greater degree with the trained leg, whereas the non-local, heterologous muscle adaptations

426 would have been more reliant upon neural adaptations (Ebersole et al. 2002; Evetovich et al.

427 2001; Farthing et al. 2007; Hortobagyi 2005; Kidgell et al. 2011; Latella et al. 2012; Lee and

428 Carroll 2007). Dynamic leg press training would not have provided substantial motor learning

429 contributions for heterologous EF or handgrip MVIC for example.

The degree of change with the muscle endurance 60\% 1RM test (104.1\% vs. $73.4 \%$ for trained and untrained legs) was substantially higher than the training changes for all other tests.

432 As this test emphasized muscle endurance rather than strength, it might suggest another

433 mechanism for cross education. Magnusson and colleagues (1996) introduced the concept of

434 stretch tolerance to explain stretch-induced increases in ROM in the absence of changes in tissue

435 compliance/stiffness and electromyography (EMG). They suggested that prior stretching or

436 stretch training would allow the individual to tolerate greater pain or discomfort and thus stretch

437 farther than before. Similarly, it could be suggested that the youth in the present study had an

438 increase in "work tolerance". The increased muscle endurance in the contralateral, untrained leg

439 cannot be ascribed to enzymatic changes. It can be argued that exposure to repeated bouts of

440 uncomfortable exertions during training allowed the youth to accommodate greater discomfort or

441 pain and push themselves past previous limits even with untrained muscles. Children's lack of

442 experience can adversely affect their perception of exertion (Williams et al. 1994; Barkley 2011;

443 Murphy et al. 2014). Whereas increased work tolerance would likely play a greater role with 
444 enduring repeated contractions $(60 \% 1 \mathrm{RM})$, it could also play a role in tolerating the discomfort

445 of higher intensity contractions and thus contributing to greater force outputs globally.

446

447 repetition group versus the low load/high repetition training. The greater effect of high intensity

448 resistance training is also reported in the adult literature (Schoenfeld et al. 2014; Mangine et al.

449 2015; Jenkins et al. 2017). In general, the literature has previously recommended that low to

450 moderate intensity resistance training should be implemented for youth (Faigenbaum et al. 1996;

451 Golan et al. 1998; McNeely et al. 2002; Malina 2006). However, the Canadian Society for

452 Exercise Physiology position stand (Behm et al. 2008) suggested that higher intensity and more

453 advanced resistance training techniques such as plyometrics and Olympic-style lifting can also

454 be employed. The higher load resistance training program would activate the full spectrum of

455 low threshold type I, and higher threshold type II (slow and fast twitch respectively) motor units

456 (Behm 1995). Other training components have also been investigated. Munn et al. (2005)

457 reported a non-significant $(\mathrm{p}=0.08)$ cross education elbow flexor training advantage with high

$458(5 \%)$ versus low $(-1 \%)$ velocity isoinertial training. As evidenced from the strength training

459 improvements in untrained ipsilateral (handgrip and EF) and contralateral (handgrip, EF, KE,

460 KF) muscles, these adaptations acted globally. Whereas the high load/low repetition training

461 provided greater strength training responses, the disparity between trained and untrained limbs

462 was lower with the low load/high repetition training group. While the untrained limbs achieved

463 mean $37.1 \%$ and $40.5 \%$ increases with high load/low repetition and low load/high repetition

464 training respectively, the trained limbs achieved 53.4\% versus $47.8 \%$ improvements with high

465 load/low repetition and low load/high repetition training respectively, which contributed to the

466 training type $\mathrm{x}$ trained limb disparity. Thus, irrespective of age (youth vs. adult), trained muscles 
467 and global cross education effects demonstrate greater strength improvements with higher 468 intensity resistance loading.

469 A major limitation of the present study was that EMG was not monitored during the 470 training program with the possibility that the non-trained leg was activated by mirror activity or

471 stabilization efforts. However, the participants were monitored by the researchers and

472 continually exhorted to relax the non-trained limb. It was beyond the scope of this study to

473 determine the precise neurological training adaptations.

474 CONCLUSIONS

Should the term cross education apply with ipsilateral and contralateral heterologous muscle training adaptations? Perhaps a more apt term would be global education or global

477 training effects. The present study is the first to demonstrate cross or global education effects 478 with children and illustrated that these effects act not only on contralateral homologous (cross 479 education) muscles but also global training effects to heterologous ipsilateral and contralateral 480 muscles. It is likely that the mechanisms for the trained muscles and contralateral homologous 481 muscles include neural adaptations (i.e. motor unit recruitment, rate coding) and motor control / 482 learning adaptations. The lower relative strength gains for the untrained EF and handgrip 483 muscles (different muscles and movements; lack of training specificity) suggest that motor 484 learning changes may not have played as much of a significant role. The greatest strength 485 training responses involved high load/low repetition training versus the low load/high repetition 486 training. As such, the extent of cross education effects and the greater effectiveness of high 487 load/low repetition training illustrates similar responses between youth and adults. However, due 
488 to the lack of adult studies examining global training effects, it is not known whether adult

489 heterologous untrained muscles would respond similarly to youth.

490

491

492

CONFLICT OF INTEREST: The authors declare no conflicts of interest, financial or 493

494

495 REFERENCES

496 Aboodarda, S.J., Copithorne, D.B., Power, K.E., Drinkwater, E., and Behm, D.G. 2015. Elbow

497 flexor fatigue modulates central excitability of the knee extensors. Appl. Physiol. Nutr. Metab.

$498 \quad$ 40: $924-30$.

499 Aboodarda, S.J., Sambaher, N., Millet, G.Y., and Behm, D.G. 2017. Knee extensors

500 neuromuscular fatigue changes the corticospinal pathway excitability in biceps brachii muscle.

501 Neurosci. 340: 477-486.

502 Adamson, M., Macquaide, N., Helgerud, J., Hoff, J., and Kemi, O.J. 2008. Unilateral arm

503 strength training improves contralateral peak force and rate of force development. Eur. J. Appl.

504 Physiol. 103: 553-9.

505 Barkley, J.E.R., Roemmich, J.N. 2011. Validity of a pediatric RPE scale when different exercise 506 intensities are completed on separate days. J. Exer. Sci. Fitness, 9: 52-57.

507 Behm, D.G. 1995. Neuromuscular implications and applications of resistance training. J.

508 Strength Cond. Res. 9: 264-274.

509 Behm, D.G., Cavanaugh, T., Quigley, P., Reid, J.C., Nardi, P.S., and Marchetti, P.H. 2016.

510 Acute bouts of upper and lower body static and dynamic stretching increase non-local joint range

511 of motion. Eur. J. Appl. Physiol. 116: 241-9. 
512 Behm, D.G., Faigenbaum, A.D., Falk, B., and Klentrou, P. 2008. Canadian Society for Exercise

513 Physiology position paper: resistance training in children and adolescents. Appl. Physiol. Nutr.

514 Metab. 33: 547-61.

515 Behm, D.G. and Sale, D.G. 1993. Velocity specificity of resistance training. Sports Med. 15:

$516 \quad 374-88$.

517 Behm, D.G.Y., J.D.; Whitten, J.H.D.; Reid, J.C.; Quigley, P.J.; Low, J.L.; Li, Y.; Lima, C.D.;

518 Hodgson, D.D.; Chaouachi, A.; Prieske, O.; Granacher, U. 2017. Effectiveness of Traditional

519 Strength versus Power Training on Muscle Strength, Power and Speed with Youth: A Systematic

520 Review and Meta-analysis. Frontiers Exerc. Physiol. June 2017

521 doi.org/10.3389/fphys.2017.00423

522 Behringer, M., Vom Heede, A., Matthews, M., and Mester, J. 2011. Effects of strength training

523 on motor performance skills in children and adolescents: a meta-analysis. Ped. Exerc. Sci. 23:

$524 \quad 186-206$.

525 Ben Othman, A., Chaouachi, A., Hammami, R., Chaouachi, M.M., Kasmi, S., and Behm, D.G.

526 2017. Evidence of nonlocal muscle fatigue in male youth. Appl. Physiol. Nutr. Metab. 42: 229-

527237.

528 Beyer, K.S., Fukuda, D.H., Boone, C.H., Wells, A.J., Townsend, J.R., Jajtner, A.R., Gonzalez,

529 A.M., Fragala, M.S., Hoffman, J.R. and Stout, J.R. 2016. Short-term unilateral resistance training 530 results in cross education of strength without changes in muscle size, activation, or endocrine

531 response. J. Strength Cond. Res. 30(5) : 1213-1223.

532 Blimkie, C.J. 1989. Age- and sex-associated variation in strength during childhood:

533 Anthropometric, morphologic, neurologic, biomechanical, endocrinologic, genetic, and physical 
534 activity correlates.In: C.V. Gisolfi (Ed.), Perspectives in Exercise Science and Sports Medicine,

535 Vol. 2: Youth, Exercise and Sports, pp. 99-163. Indianapolis, IN: Benchmark Press.

536 Carroll, T., Herbert, R., Munn, J., Lee, M., and Gandevia, S. 2006. Contralateral effects of

537 unilateral strength training: evidence and possible mechanisms. J. Appl. Physiol. 101, 15145381522.

539 Chaouachi, A., Hammami, R., Kaabi, S., Chamari, K., Drinkwater, E.J., and Behm, D.G. 2014.

540 Olympic weightlifting and plyometric training with children provides similar or greater

541 performance improvements than traditional resistance training. J. Strength Cond. Res. 28: 1483-

54296.

543 Chaouachi, M., Granacher, U., Makhlouf, I., Hammami, R., Behm, D.G., and Chaouachi, A.

544 2017. Within Session Sequence of Balance and Plyometric Exercises Does Not Affect Training 545 Adaptations with Youth Soccer Athletes. J. Sports Sci. Med. 16: 125-136.

546 Cirer-Sastre, R.B.-G., J.V.; Corbi, F. 2017. Contralateral Effects after Unilateral Strength

547 Training: A Meta-Analysis Comparing Training Loads. J. Sports Sci. Med. 16: 180-186.

548 Cohen, J. 1988. Statistical power analysis for the behavioural sciences. Hillside N.J.: L. Erbraum 549 Associates.

550 Ebersole, K.T., Housh, T., Johnson, G.O., Perry, S.R., Bull, A.J., and Cramer, J.T. 2002.

551 Mechanomyographic and electromyographic responses to unilateral isometric training. J.

552 Strength Cond. Res. 16: 192-201.

553 Evetovich, T.K., Housh, D.J., Housh, T.J., Johnson, G.O., Smith, D.B., and Ebersole, K.T. 2001.

554 The effect of concentric isokinetic ctrength training of the quadriceps femoris on

555 electromyography and muscle strength in the trained and untrained limb. J. Strength Cond. Res.

556 15: 439-445. 
557 Faigenbaum, A. 2000. Strength training for children and adolescents. Pediatric Adolescent

$558 \quad$ Sports Injuries, 19: 593-619.

559 Faigenbaum, A.D., Kraemer, W.J., Blimkie, C.J., Jeffreys, I., Micheli, L.J., Nitka, M., and

560 Rowland, T.W. 2009. Youth resistance training: updated position statement paper from the 561 national strength and conditioning association. J. Strength Cond. Res. 23: S60-S79.

562 Faigenbaum, A.D., Kraemer, W.J., Cahill, B., Chandler, J., Dziados, J., Elfrink, L.D., Formann, 563 E., Gaudiose, M., Micheli, L., Nitka, M., and Roberts, S. 1996. Youth resistance training:

564 Position statement paper and literature review. Strength Cond. December: 62-75.

565 Faigenbaum, A.D., Milliken, L.A., and Westcott, W.L. 2003. Maximal strength testing in healthy 566 children. J. Strength Cond. Res. 17: 162-166.

567 Faigenbaum, A.D., Westcott, W.L., Loud, R.L., and Long, C. 1999. The effects of different 568 resistance training protocols on muscular strength and endurance development in children.

569 Pediatrics, 104: e5.

570 Faigenbaum, A.D.W., Michelli, LJ. (1996). The effects of strength training and detraining on

571 children. J. Strength Cond. Res. 10: 109-114.

572 Falk, B. and Tenenbaum, G. 1996. The effectiveness of resistance training in children. A meta573 analysis. Sports Med. 22: 176-86.

574 Farthing, J.P. 2009. Cross-education of strength depends on limb dominance: implications for 575 theory and application. Exerc. Sport Sci. Rev. 37: 179-87.

576 Farthing, J.P., Borowsky, R., Chilibeck, P.D., Binsted, G., and Sarty, G.E. 2007. Neuro-

577 physiological adaptations associated with cross-education of strength. Brain Topogr. 20: 77-88.

578 Farthing, J.P. and Chilibeck, P.D. 2003. The effect of eccentric training at different velocities on 579 cross-education. Eur. J. Appl. Physiol. 89: 570-7. 
580 Farthing, J.P., Chilibeck, P.D., and Binsted, G. 2005. Cross-education of arm muscular strength 581 is unidirectional in right-handed individuals. Med. Sci. Sports Exerc. 37: 1594-1600.

582 Golan, R., Falk, B., Hoffman, J., Hochberg, Z., Ben-Sira, D., and Barak, Y. 1998. Resistance 583 training for children and adolescents. Position Statement for the International Federation of 584 Sports Medicine: 265-270.

585 Goodwill A.M., and Kidgell, D.J. 2012. The Effects of Whole-Body Vibration on the Cross586 Transfer of Strength. Scientific World Journal 2012: 1-11, 2012 doi:10.1100/2012/504837

587 Halperin, I., Aboodarda, S.J., and Behm, D.G. 2014. Knee extension fatigue attenuates repeated 588 force production of the elbow flexors. Eur. J. Sport Sci. 14: 823-829.

589 Hortobagyi, T. 2005. Cross Education and the Human Central Nervous System: Mechanisms of 590 Unilateral Interventions Producing Contralateral Adaptations. IEEE Eng Med. Biol.

591 January/February: 22-28.

592 Hortobagyi, T., Lambert, N., Tracy, C., and Shinebarger, M. 1992. Voluntary and 593 electromyostimulation forces in trained and untrained men. Med. Sci. Sports Exerc. 24: 702-707. 594 Hortobagyi, T., Lambert, N.J., and Hill, J.P. 1997. Greater cross education following training 595 with muscle lengthening than shortening. Med. Sci. Sports Exerc. 29: 107-12.

596 Housh, D., Housh, T., Johnson, G., and Chu, W. 1992. Hypertrophic response to unilateral 597 concentric isokinetic resistance training. J. Appl. Physiol. 73: 65-70.

598 Housh, D.J., Housch, T.J., Weir, J.P., Weir, L.L., Evetovich, T.K., and Donlin, P.E. 1998.

599 Effects of unilateral concentric-only dynamic constant external resistance training on quadriceps 600 femoris cross-sectional area. J. Strength Cond. Res. 12: 185-191.

601 Housh, D.J. and Housh, T.J. 1993. The effects of unilateral velocity-specific concentric strength 602 training. J. Orthop. Sports Phys. Ther. 17: 252-6. 
603 Jenkins N.D.M., Miramonti A.A., Hill E.C., Smith C.M., Cochrane-Snyman K.C., Housh T.J. 604 and Cramer J.T. 2017. Greater Neural Adaptations following High- vs. Low-Load Resistance 605 Training. Front. Physiol. 8:331. doi: 10.3389/fphys.2017.00331

606 Kannus, P., Alosa, D., Cook, L., Johnson, R.J., Renstrîm, P., Pope, M., Beynnon, B., Nichols, C., 607 and Kaplan, M. 1992. Effect of one-legged exercise on the strength, power and endurance of the 608 contralateral leg. A randomized, controlled study using isometric and concentric isokinetic 609 training. Eur. J. Appl. Physiol. 64: 117-126.

610 Kidgell, D.J., Stokes, M.A., and Pearce, A.J. 2011. Strength Training of One Limb Increases 611 Corticomotor Excitability Projecting to the Contralateral Homologous Limb. Motor Control, 15, $612 \quad 247-266$

613 Latella, C., Kidgell, D.J., and Pearce, A.J. 2012. Reduction in corticospinal inhibition in the 614 trained and untrained limb following unilateral leg strength training. Eur. J. Appl. Physiol. 112: $615 \quad 3097-107$.

616 Lee, M. and Carroll, T.J. 2007. Cross education: possible mechanisms for the contralateral 617 effects of unilateral resistance training. Sports Med. 37: 1-14.

618 Magnusson, S.P., Simonsen, E.B., Aagaard, P., Sorensen, H., and Kjaer, M. 1996. A mechanism 619 for altered flexibility in human skeletal muscle. J. Physiol. 497 ( Pt 1): 291-298.

620 Malina, R. 2006. Weight training in youth. Growth, maturation and safety: An evidence based 621 review. Clin. J. Sports Med. 16: 478-487.

622 Mangine G.T., Hoffman J.R., Gonzalez A.M., Townsend J.R., Wells A.J., Jajtner A.R., Beyer 623 K.S., Boone C.H., Miramonti A.A., Wang R, LaMonica .B., Fukuda D.H., Ratamess N.A. and 624 Stout J.R. 2015. The effect of training volume and intensity on improvements in muscular 625 strength and size in resistance-trained men. Physiol Reports, 3(8) : e12472, doi: 
626

627

628

629

630

631

632

633

634

635

636

637

638

639

640

641

642

643

644

645 Cooper Publishing Group.

646 Sambaher, N., Aboodarda, S.J., and Behm, D.G. 2016. Bilateral Knee Extensor Fatigue

647 Modulates Force and Responsiveness of the Corticospinal Pathway in the Non-fatigued,

648 Dominant Elbow Flexors. Front. Hum. Neurosci. 10: 18-24. 
649 Sariyildiz, M., Karacan, I., Rezvani, A., Ergin, O., and Cidem, M. 2011. Cross-education of 650 muscle strength: cross-training effects are not confined to untrained contralateral homologous

651 muscle. Scand. J. Med. Sci. Sports, 21: e359-64.

652 Schoenfeld B.J., Wilson J.M., Lowery R.P., and Krieger J.W. 2014. Muscular adaptations in 653 low- versus high-load resistance training: A meta-analysis. Eur. J. Sport Sci.

654 http://dx.doi.org/10.1080/17461391.2014.989922

655 Scripture, E.W.S. 1894. On the education of muscular control and power. Studies of the Yale 656 Psychology Laboratory, 2: 114-119.

657 Shields, R.K., Leo, K.C., Messaros, A.J., and Somers, V.K. 1999. Effects of repetitive handgrip 658 training on endurance, specificity, and cross-education. Phys. Ther. 79: 467-75.

659 Williams, J.G., Eston, R., and Furlong, B. 1994. CERT - a perceived exertion scale for young660 children. Perceptual Motor Skills, 79: 1451-1458.

661

662

663

664

665

666

667

668

669

670

671

672 
Table 1: Participant characteristics. Acronyms: BMI: Body mass index, PHV: peak height velocity

\begin{tabular}{ccccccc}
\hline Groups & $\begin{array}{c}\text { Age } \\
\text { (years) }\end{array}$ & $\begin{array}{c}\text { Mass } \\
(\mathbf{K g})\end{array}$ & $\begin{array}{c}\text { Height } \\
(\mathbf{c m})\end{array}$ & BMI & $\begin{array}{c}\text { Body Fat } \\
\text { \% }\end{array}$ & PHV \\
N=16 for each group & & & & & & \\
\hline High load-low repetition & $11.8 \pm 0.6$ & $40.1 \pm 3$ & $147.5 \pm 5.5$ & $18.5 \pm 1.6$ & $17.9 \pm 3.3$ & $-2.7 \pm 0.3$ \\
Low load-high repetition & $11.5 \pm 0.5$ & $38.3 \pm 3.5$ & $147.5 \pm 4.7$ & $17.7 \pm 1.8$ & $18.4 \pm 2.8$ & $-2.8 \pm 0.3$ \\
Control & $11.9 \pm 1.2$ & $38.8 \pm 8.1$ & $146 \pm 3.5$ & $18.2 \pm 1.2$ & $18.5 \pm 2.8$ & $-2.5 \pm 1.6$ \\
\hline
\end{tabular}

680

681

682

683

684

685

686

687

688

689

690

691

692

693 
Table 2: Reliability: Intraclass Correlation Coefficients Abbreviations: KE: knee extension, KF: knee flexion, EF: elbow flexion, $\mathrm{CMJ}$ : countermovement jump, 1RM: 1 repetition maximum, rep: repetition

\begin{tabular}{|l|l|l|l|l|l|l|l|}
\hline & Handgrip & KE MVIC & KF MVIC & EF MVIC & CMJ & $\begin{array}{l}\text { 1RM Leg } \\
\text { Press }\end{array}$ & $\begin{array}{l}60 \% \text { 1RM } \\
\text { Leg Press }\end{array}$ \\
\hline $\begin{array}{l}\text { Low Load } \\
\text { High Rep }\end{array}$ & 0.908 & 0.908 & 0.759 & 0.919 & 0.951 & 0.982 & 0.827 \\
\hline $\begin{array}{l}\text { High Load } \\
\text { Low Rep }\end{array}$ & 0.782 & 0.792 & 0.732 & 0.835 & 0.882 & 0.975 & 0.861 \\
\hline Control & 0.754 & 0.754 & 0.883 & 0.876 & 0.821 & 0.948 & 0.948 \\
\hline
\end{tabular}


Table 3: The adjusted magnitude of contralateral and global strength transfer effects between groups. Absolute mean percentage differences are compared to contralateral or global strength transfer (GST) calculations. Asterisks illustrate significant differences calculated from absolute mean values. Acronyms: HL-LR: High load - low repetition, LL-HR: Low load - high repetition, MVIC: maximum voluntary isometric contraction, $\mathrm{CMJ}$ : countermovement jump, 1RM: 1 repetition maximum.

\begin{tabular}{|c|c|c|c|c|c|c|}
\hline & HL-LR vs & ontrol & LL-HR v & ntrol & HL-LR v & L-HR \\
\hline & Absolute & GST & Absolute & GST & Absolute & GST \\
\hline Handgrip MVIC & $17.6 \% *$ & $3.7 \%$ & $10.4 \%$ & $11.1 \%$ & $-8.6 \%$ & $-7.4 \%$ \\
\hline Knee Extension MVIC & $24.3 \% *$ & $55.1 \%$ & $18.1 \% *$ & $27.9 \%$ & $25.5 \% *$ & $27.2 \%$ \\
\hline Knee Flexion MVIC & $20.9 \% *$ & $31.8 \%$ & $11.7 \% *$ & $16.3 \%$ & $20.0 \% *$ & $15.3 \%$ \\
\hline Elbow Flexion MVIC & $11.2 \% *$ & $12.3 \%$ & $4.6 \%$ & $1.8 \%$ & $10.8 \% *$ & $10.4 \%$ \\
\hline CMJ & $19.1 \% *$ & $20.5 \%$ & $5.3 \% *$ & $26.8 \%$ & $-14.8 \%$ & $-6.3 \%$ \\
\hline Leg Press 1RM & $25.6 \% *$ & $44.9 \%$ & $20.9 \%$ & $23.3 \%$ & $31.6 \% *$ & $21.7 \%$ \\
\hline Leg press $60 \% 1 \mathrm{RM}$ & $44.1 \% *$ & $69.4 \%$ & $57.2 \% *$ & $122.7 \%$ & $-33.3 \%$ & $-53.4 \%$ \\
\hline
\end{tabular}




\section{FIGURE LEGENDS}

\section{Figure 1: Knee extensors MVIC}

Acronyms: LL-HR: low load high repetitions, HL-LR: high load low repetitions HR represented by grey lines or dots, LR represented by black lines or hyphens and control represented by closed lines. Asterisk $(*)$ indicates that HL-LR group was significantly greater than control at post-test. Hash tag or number sign (\#) indicates HL-LR significantly exceeded LL-HR at post-test.

Figure 2: Figure 2: Knee Flexors MVIC Acronyms: LL-HR: low load high repetitions, HL-LR: high load low repetitions HR represented by grey lines or dots, LR represented by black lines or hyphens and control represented by closed lines. Asterisk (*) indicates that HL-LR group was significantly greater than control at post-test. Hash tag or number sign (\#) indicates HL-LR significantly exceeded LL-HR at post-test.

Figure 3: Countermovement Jump Height (CMJ)

Acronyms: LL-HR: low load high repetitions, HL-LR: high load low repetitions HR represented by grey lines or dots, LR represented by black lines or hyphens and control represented by closed lines. Asterisk (*) indicates that HL-LR group was significantly greater than control at post-test.

Figure 4: Figure 4: 1Repetition Maximum (RM) Leg Press

Acronyms: LL-HR: low load high repetitions, HL-LR: high load low repetitions HR represented by grey lines or dots, LR represented by black lines or hyphens and control represented by closed lines. Asterisk $\left(^{*}\right)$ indicates that HL-LR group was significantly greater than control at post-test. Hash tag or number sign (\#) indicates HL-LR significantly exceeded LL-HR at post-test.

Figure 5: 60\% of 1RM Leg Press Acronyms: LL-HR: low load high repetitions, HL-LR: high load low repetitions HR represented by grey lines or dots, LR represented by black lines or hyphens and control represented by closed lines. Asterisk (*) indicates that HL-LR group was significantly greater than control at post-test.

Figure 6: Handgrip Strength HR represented by grey lines or dots, LR represented by black lines or hyphens and control represented by closed lines. Asterisk $\left(^{*}\right)$ indicates that HL-LR group was significantly greater than control at post-test. 
776

777 Figure 7: Elbow Flexors MVIC

778 Acronyms: LL-HR: low load high repetitions, HL-LR: high load low repetitions, IPSI: Ipsilateral

779

780

781

782

783 arm to the trained leg, CONTRA: contralateral arm to the trained leg HR represented by grey lines or dots, LR represented by black lines or hyphens and control represented by closed lines. Asterisk $(*)$ indicates that HL-LR group was significantly greater than control at post-test. Hash tag or number sign (\#) indicates HL-LR significantly exceeded LL-HR at post-test. 
Figure 1:

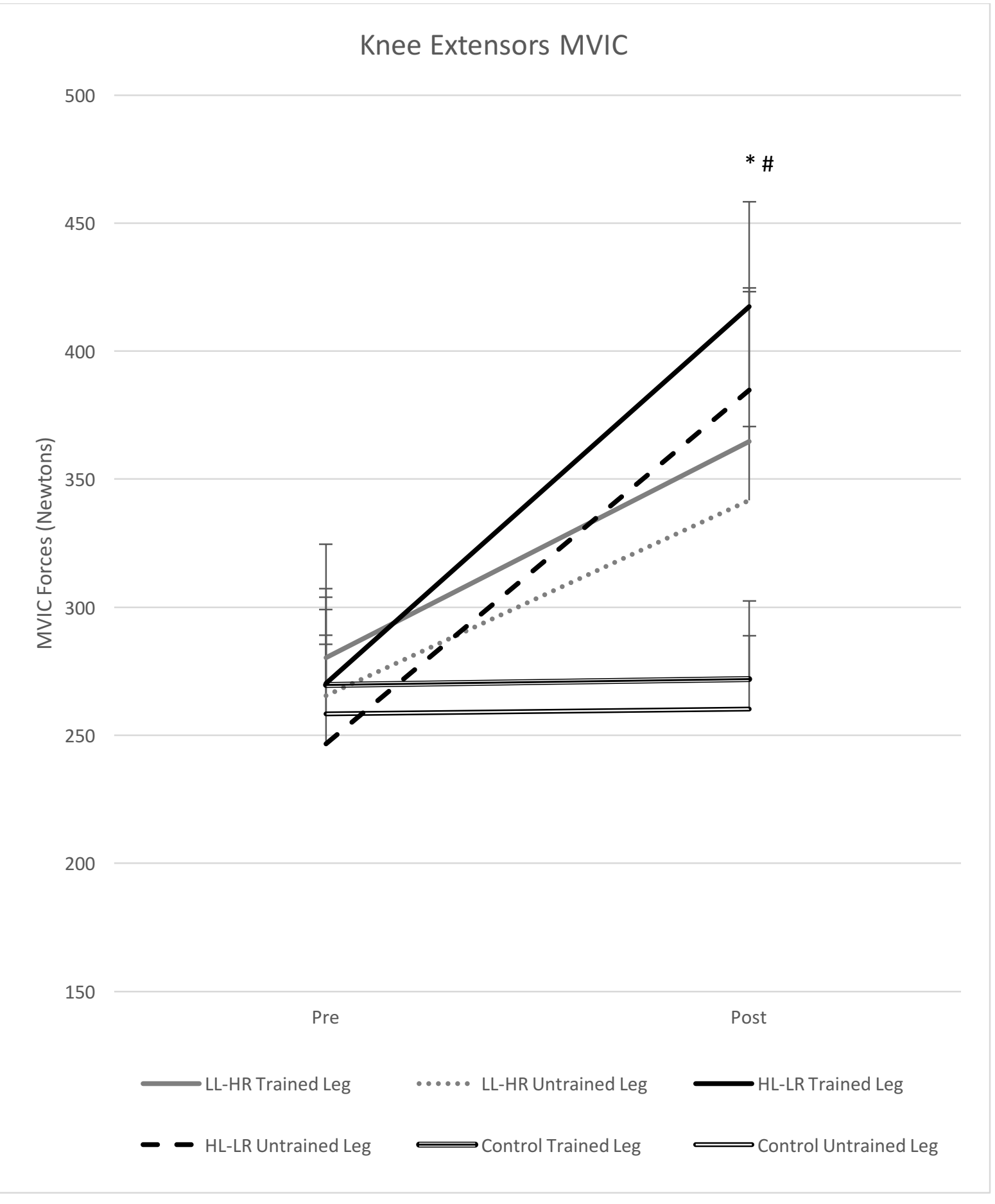


Figure 2:

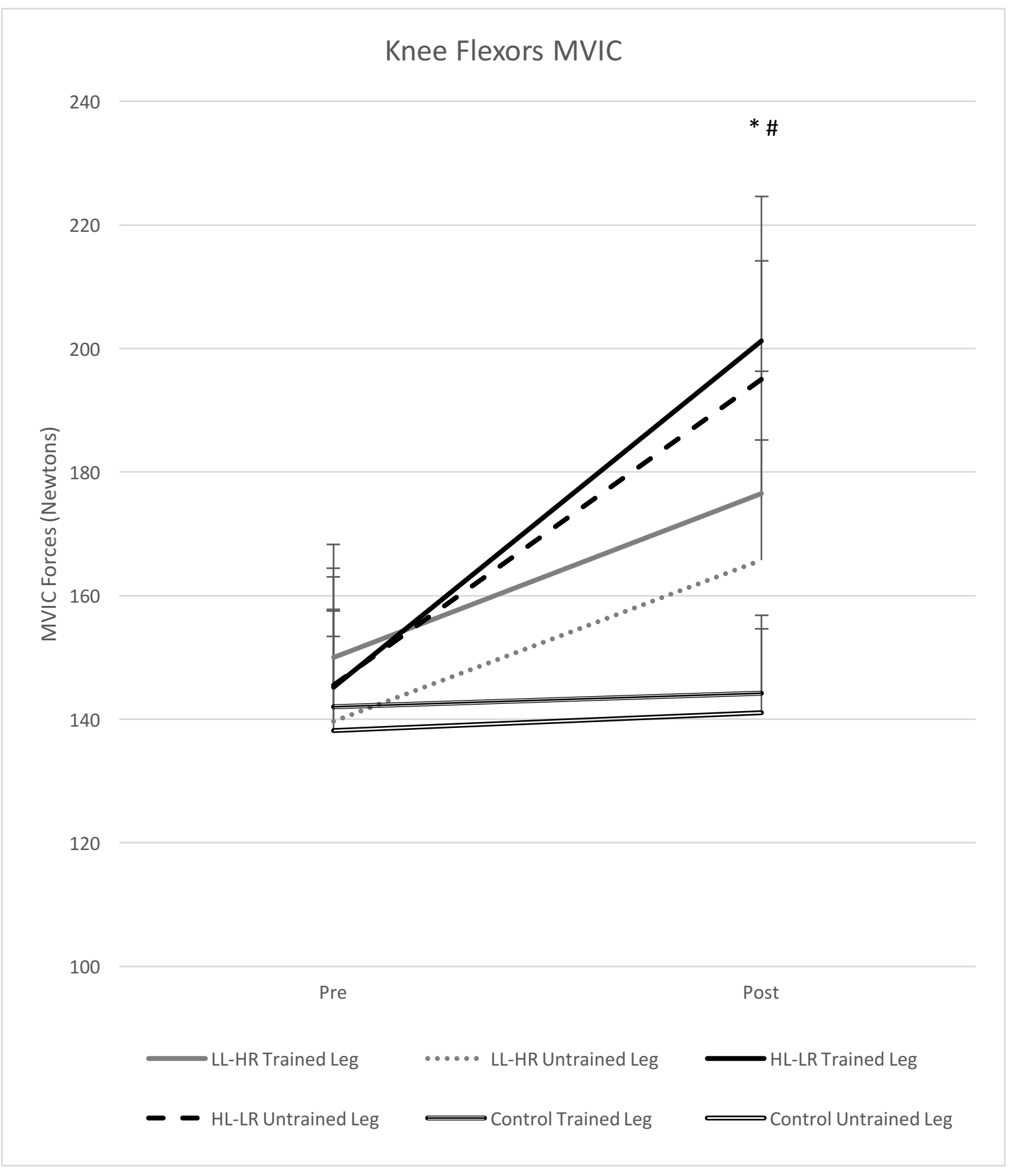


Figure 3:

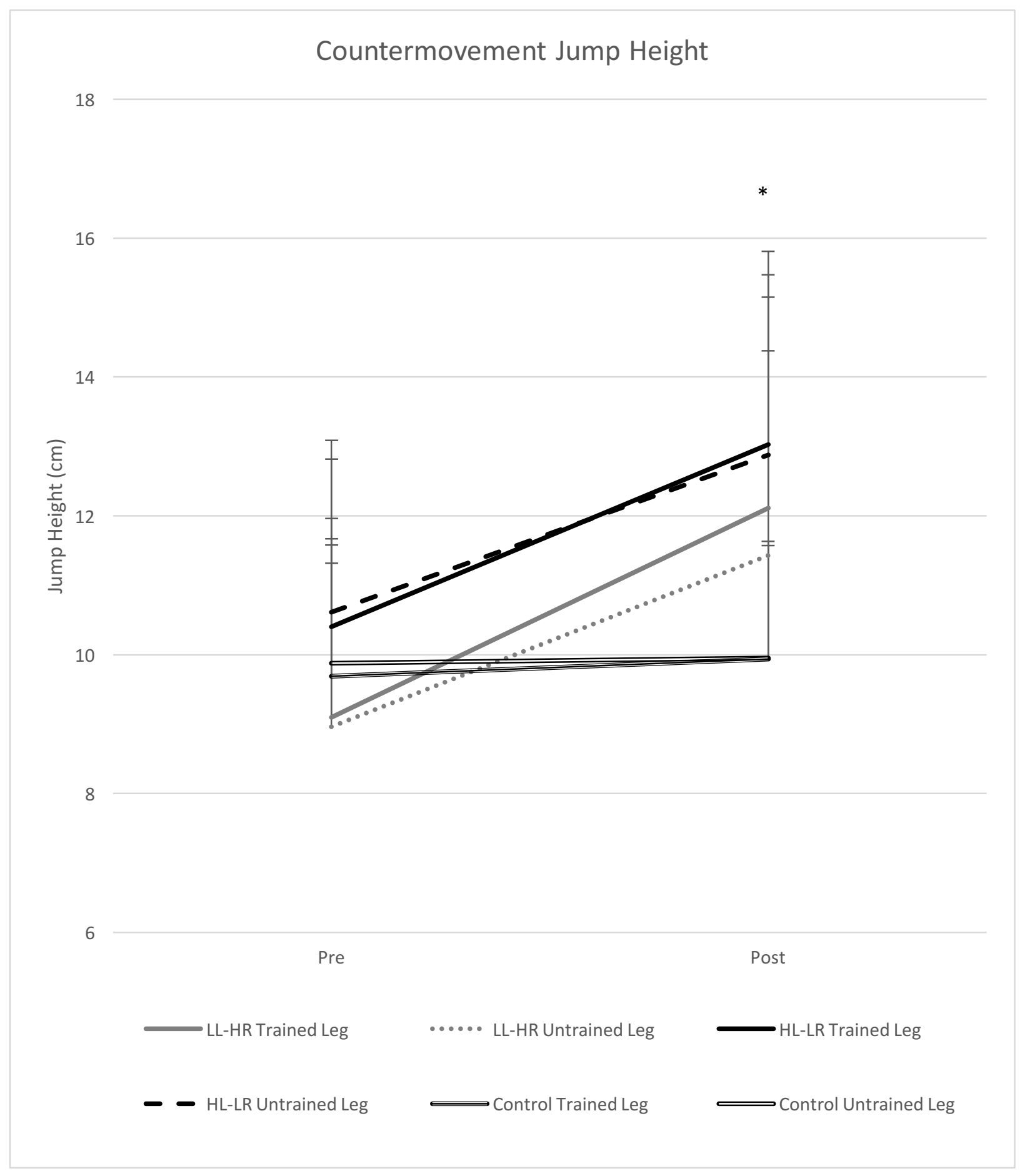


Figure 4:

\section{RM Leg Press}

70

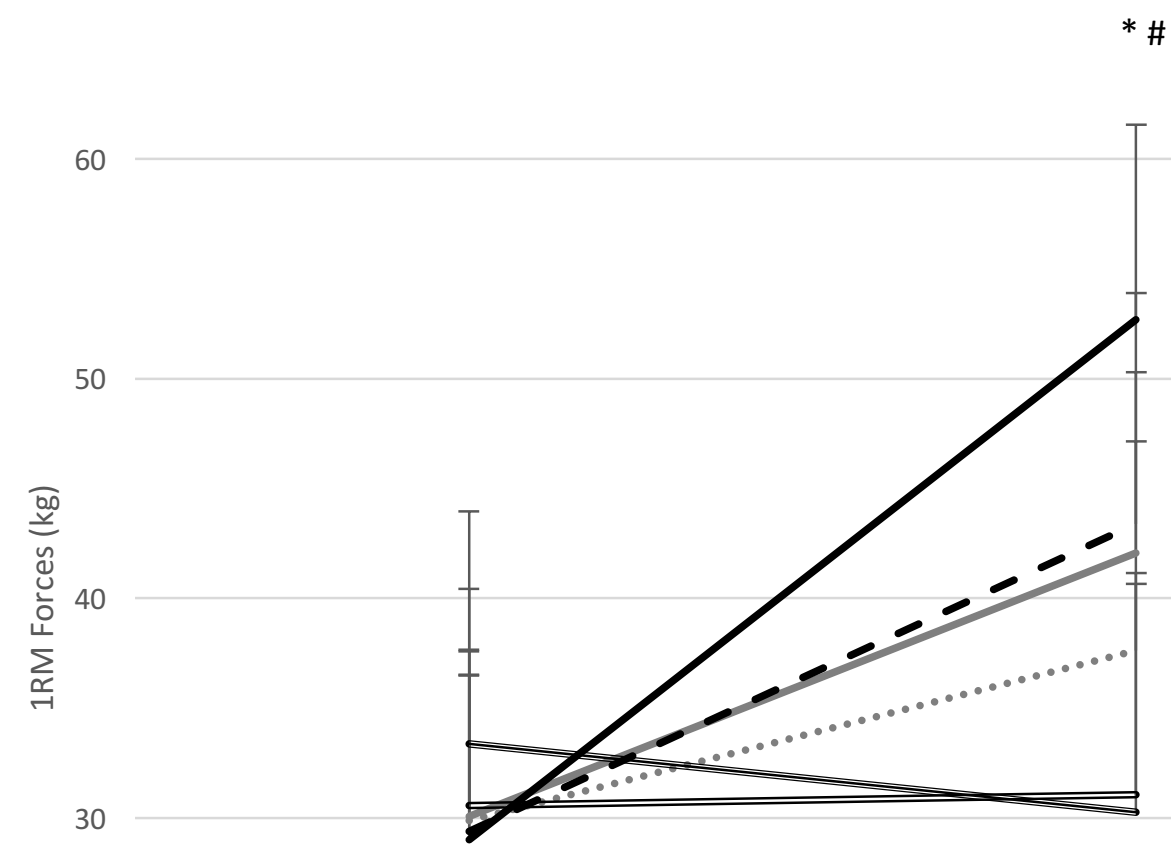

20

10

Pre

LL-HR Trained Leg

- - HL-LR Untrained Leg

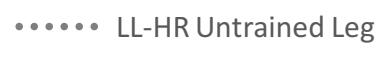

Control Trained Leg
Post

HL-LR Trained Leg

Control Untrained Leg 
Figure 5:

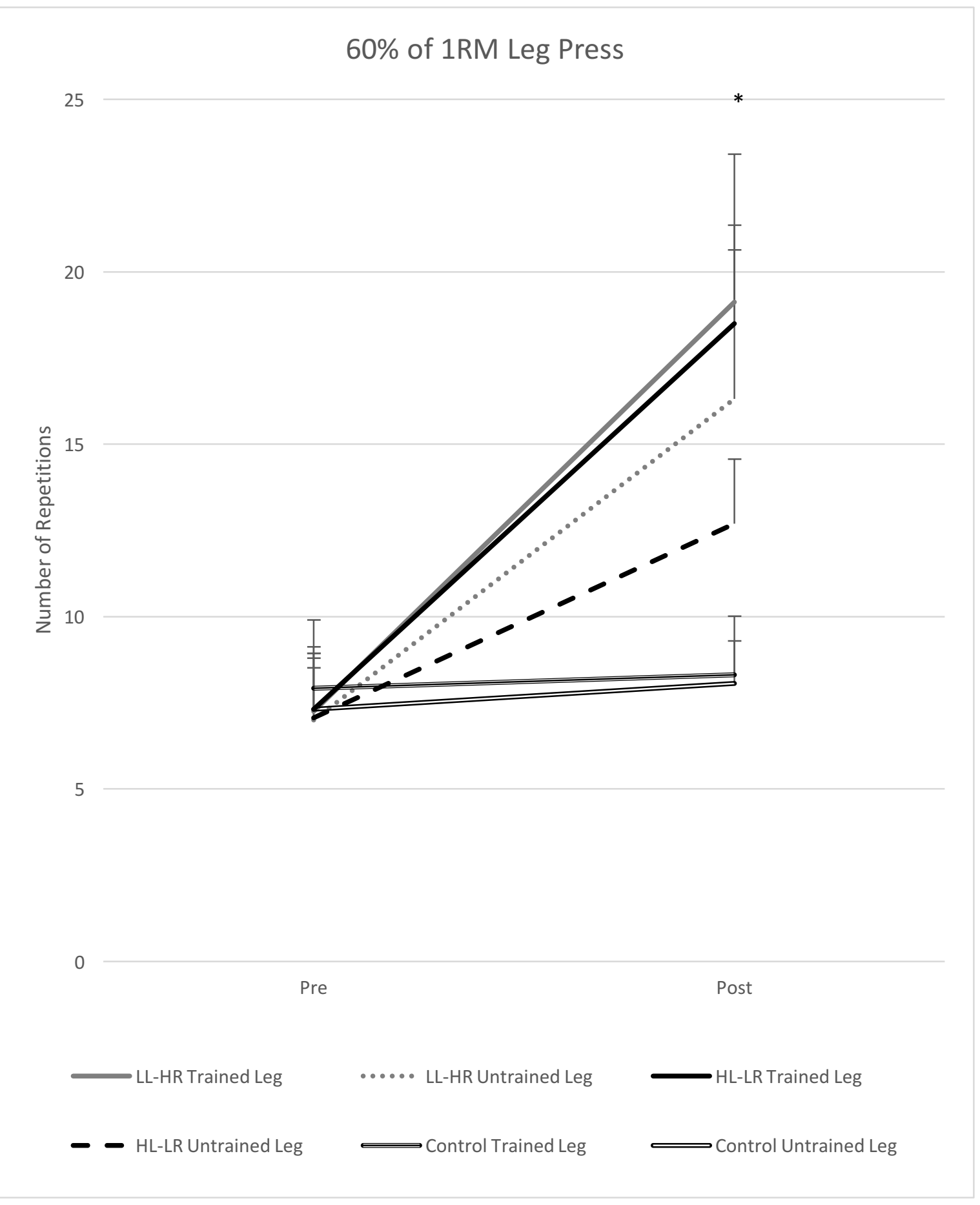


Figure 6:

\section{Handgrip Strength}

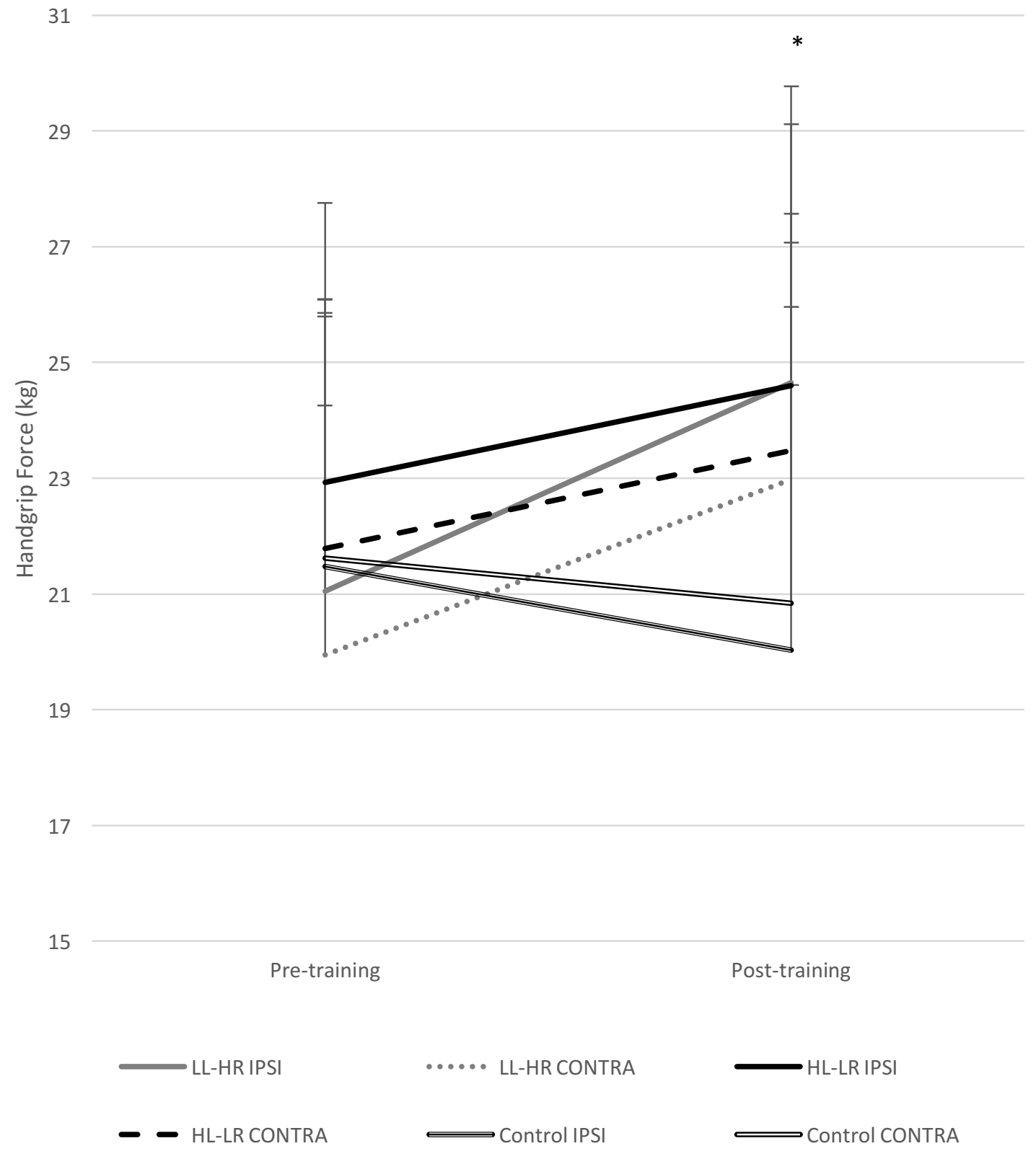


Figure 7:

\section{Elbow Flexors MVIC}

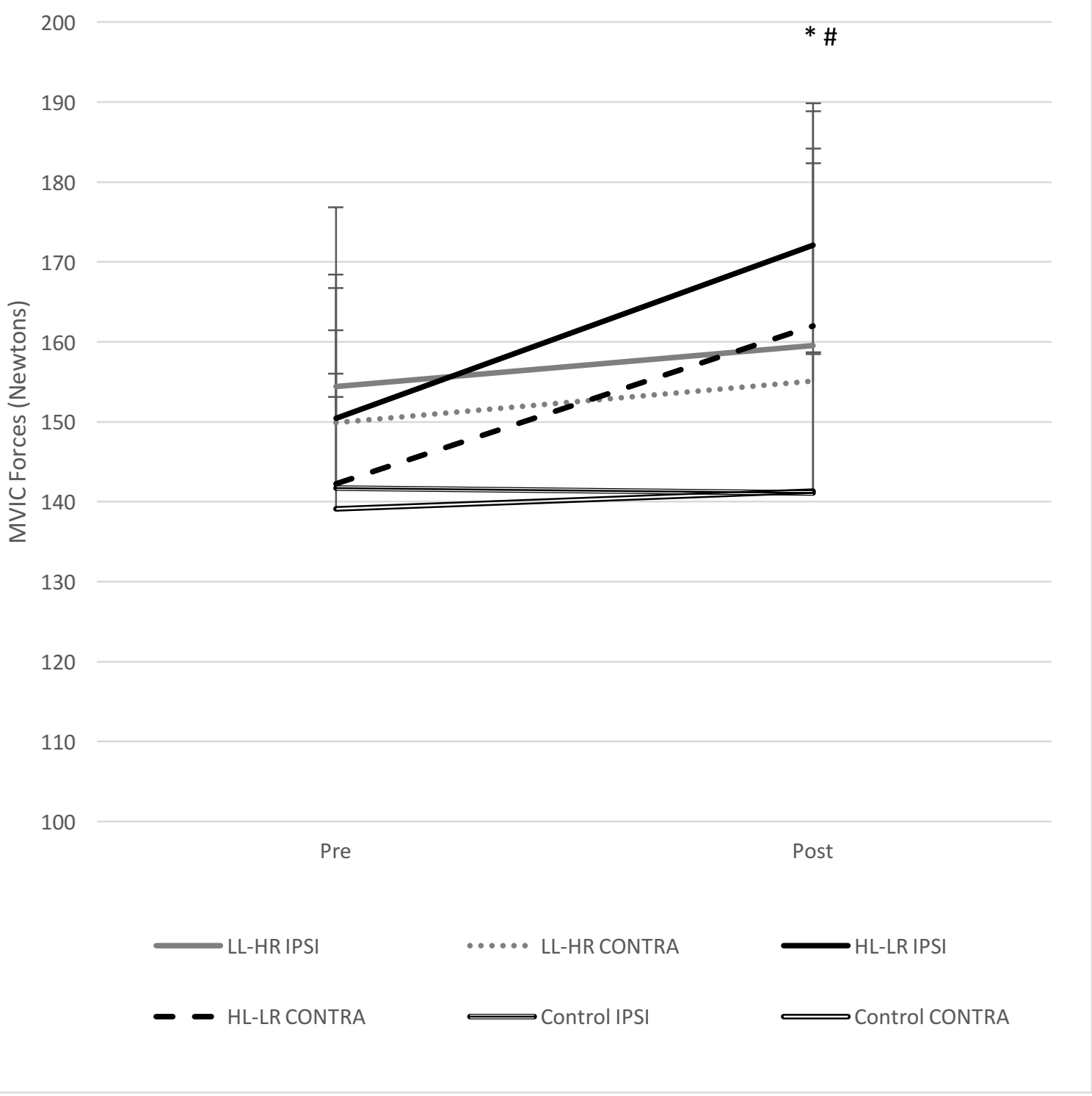

